\title{
Solution of Finite Difference Method and Differential Quadrature Method in Burgers Equation
}

\author{
Amiruddin Ab. Aziz, ${ }^{\text {a* }}$ Noor Syazana Ngarisan, ${ }^{a}$ and Nur Afriza Baki, ${ }^{a}$ \\ a) Faculty of Computer and Mathematical Sciences, Universiti Teknologi MARA, Malaysia \\ *Corresponding author: amiru2830@tganu.uitm.edu.my
}

\section{Paper History}

Received: 16-November-2018

Received in revised form: 10-November-2019

Accepted: 18-November-2019

\begin{abstract}
The Finite Difference Method and Differential Quadrature Method are used to solve the partial differential equation in Burgers equation. FDM is easy to solve but the problem is less accurate. Then, the DQM is used to overcome the problem but it needs more calculation and more time. The different number of nodes is used in these methods to investigate the accuracy. The solutions of these methods are compared in terms of accuracy of the numerical solution. $\mathrm{C}$ language program have been developed based on the method in order to solve the Burgers equation. The results of this study are compared in terms of convergence as well as accuracy of the numerical solution. Generally, from the numerical results show that the Differential Quadrature Method is better than the Finite Different Method in terms of accuracy and convergence.
\end{abstract}

KEY WORDS: Finite Difference Method (FDM), Differential Quadrature Method (DQM), Heat Transfer.

\subsection{INTRODUCTION}

Single or a system of Partial Differential Equations (PDEs) is mostly encountered by us in many sciences and engineering fields such as heat transfer [1]. PDEs also describe many of the basic natural laws in physical or chemical phenomena. Nonlinear partial differential equations are important in most fields of science. Burgers-Huxley equation has a great significance to be a nonlinear partial differential equation [2]. In this study, the partial differential equation considered is the Burgers-Huxley equations, which can effectively models the interaction between diffusion transports, convection effects and reaction mechanisms [3]. In general, the closed-form solution is not available or not easily obtained because most of these problems may involve the nonlinear partial differential equations. This fact leads to the development of another alternative to approximate the solutions of these partial differential equations. Consequently, after years of scientific research found that the approximation of the system of partial differential equations can be obtained by using numerical discretization technique at some function value at certain discrete points, called grid points or mesh points. There are three numerical methods most commonly used in engineering and in the dynamics of computing fluid are finite differences, finite element, and finite volume method.

The Burgers-Huxley is a nonlinear partial differential equation (PDE) which is important in modeling various mechanisms in engineering field such as the nonlinear wave process in economics, physics and ecology [4]. There is no general numerical approximation to obtain the solution for this non-linear PDE according to many researchers. They have adopted different numerical techniques in approximating the solution of the Burgers-Huxley equation. However, Yefimova \& Kudryashov (2004) and Gao \& Zhao (2009) have been solved the exact solution for the Burgers-Huxley equation $[5,6]$.

One of the techniques to solve the Burgers equation is to use the Finite Difference Method (FDM) method which is the simplest method. This method is solved by replacing the value at a particular grid point and approaching the derivative with the difference in these values. Partial derivatives in PDE at each grid point are estimated from the neighborhood value.

Another technique which is discussed in this study is Differential Quadrature Method (DQM). As stated by [7] DQM is an extension of FDM for the highest order of finite difference scheme. This method represents by sum up all the derivatives of the function at any grid points, and then the equation transforms to a system of ordinary differential equations (ODEs) or a set of algebraic equations [8]. Lastly, the system of ordinary differential equations is solved by using numerical methods.

There are many ways to approximate the solution of Burgers equation. A set of initial and boundary conditions are needed to solve the Burgers equation. FDM is easy to solve the examples of 
Burgers equation but is less accurate. Then, the DQM is used to solve the problem but it needs more calculation and more time consuming.

In the 1960s and 1970s the FDM have dominated in computational sciences since its inception and were the method of choice in that year. The characteristics of the finite difference method are this method utilizes uniformly spaced grids. Other characteristic is at each node, each derivative is approximated by an algebraic expression which references the adjacent nodes. Another of two characteristics are a system of algebraic equations is obtained by evaluating the previous step for each node and the system is solved for the dependent variable. FDM is used to approximate the derivative of a function values at one point by using the function value at discrete points. The solution of the PDE or ODE can be approximated by substituting the derivatives expression with the FDM. This method is easy to be applied but it is less accurate compare to DQM.

In the year 1972, to overcome the problem from FDM, the DQM was first proposed by R.E. Bellman and his associates. This method important in many fields such as biosciences, system identification, diffusion, transport process, fluid dynamics [9], chemical engineering, lubrication, acoustics and contact problem [10]. DQM has been shown to be equivalent to the general collocation method [11]. According to Shu \& Chew (1998), it is also equivalent to the highest order finite difference scheme, which is the extension of the low order finite difference schemes [12].

The DQM is used to convert the partial differential equation to ordinary differential equation by replacing the derivative of a smooth function with a weighted linear combination value [13]. This method used the function values on all grid points, so that this method is more expensive and increase the time consumed to get the solution.

\subsection{METHODOLOGY}

\section{Burgers-Huxley Equation}

Sari \& Gurarslan (2009) [3] stated that the generalized BurgersHuxley equation can be used to model interaction between diffusion transports, convection effects and reaction mechanisms are as follows [1]:

$$
\frac{\partial u}{\partial t}-\varepsilon \frac{\partial^{2} u}{\partial x^{2}}+\alpha u^{\delta} \frac{\partial u}{\partial x}=\beta\left(1-u^{\delta}\right)\left(u^{\delta}-\gamma\right) u
$$

where $\alpha, \beta, \gamma$, and $\delta$ are parameters that $\beta \geq 0, \delta>0$, with initial condition,

$$
u(x, 0)=\left(\frac{\gamma}{2}+\frac{\gamma}{2} \tanh \left(A_{1} x\right)\right)^{\frac{1}{\delta}}
$$

and the boundary conditions,

$$
\begin{aligned}
& u(0, t)=\left(\frac{\gamma}{2}+\frac{\gamma}{2} \tanh \left(-A_{1} A_{2} x\right)\right)^{\frac{1}{\delta}} \quad t \geq 0 \\
& u(1, t)=\left(\frac{\gamma}{2}+\frac{\gamma}{2} \tanh \left(A_{1}\left(1-A_{2} t\right)\right)\right)^{\frac{1}{\delta}} \quad t \geq 0
\end{aligned}
$$

where,

$$
\begin{aligned}
& A_{1}=\frac{-\alpha \delta+\delta \sqrt{\alpha^{2}+4 \beta(1+\delta)}}{4(1+\delta)} \gamma \\
& A_{2}=\frac{\gamma \alpha}{(1+\delta)}+\frac{(1+\delta-\gamma)\left(-\alpha+\sqrt{\alpha^{2}+4 \beta(1+\delta)}\right)}{2(1+\delta)}
\end{aligned}
$$

with Dirichlet boundary conditions

$$
u(a, t)=f(t) \text { and } u(b, t)=g(t)
$$

The example of the non-linear Burgers equation is given by

$$
\frac{\partial u}{\partial t}+u \frac{\partial u}{\partial x}=0 \quad 0<x<1, t>0
$$

with the initial condition

$$
u(x, 0)=x \quad 0<x<1
$$

and the boundary equations

$$
u(0, t)=0 \quad t>0
$$

\subsection{RESULTS AND DISCUSSION}

The example above has been solved by using $\mathrm{C}++$ programming with different methods.

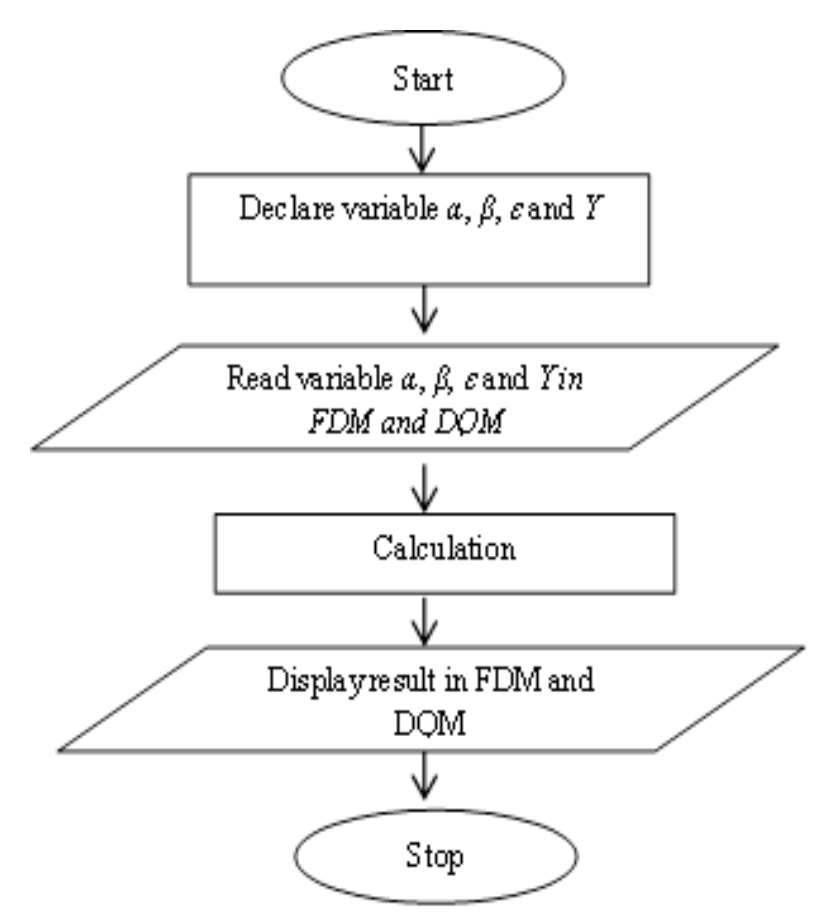

Figure 1: Flowchart of $\mathrm{C}++$ programming 
The results from FDM and DQM are compared in term of accuracy of the exact solution for the time $t=0.04$ and 0.08 .

Table 1: The results for time, $t=0.04$

\begin{tabular}{|c|c|c|c|c|c|c|c|}
\hline \multicolumn{2}{|c|}{$u(x, t) \backslash x$} & $\mathbf{0}$ & 0.2 & 0.4 & 0.6 & 0.8 & 1 \\
\hline \multirow{2}{*}{\multicolumn{2}{|c|}{ FDM }} & 0 & 0.1923 & 0.3846 & 0.5769 & 0.7692 & 1 \\
\hline & & & 1 & 2 & 3 & 4 & \\
\hline \multirow{2}{*}{\multicolumn{2}{|c|}{ DQM }} & 0 & 0.1923 & 0.3846 & 0.5769 & 0.7692 & 1 \\
\hline & & & 08 & 15 & 23 & 31 & \\
\hline \multirow{2}{*}{\multicolumn{2}{|c|}{ EXACT }} & 0 & 0.1923 & 0.3846 & 0.5769 & 0.7692 & 0.9615 \\
\hline & & & 07251 & 14503 & 21754 & 29006 & 36257 \\
\hline \multirow{4}{*}{$\begin{array}{l}\text { ER } \\
\text { RO } \\
\text { RS }\end{array}$} & \multirow{2}{*}{ FDM } & 0 & 2.7485 & 5.4970 & 8.2455 & 1.0994 & 0.0384 \\
\hline & & & $1 \mathrm{E}-06$ & $1 \mathrm{E}-06$ & $2 \mathrm{E}-06$ & E-05 & 63743 \\
\hline & \multirow{2}{*}{ DQM } & 0 & 7.4850 & 4.9701 & 1.2455 & 1.9940 & 0.0384 \\
\hline & & & $6 \mathrm{E}-07$ & $2 \mathrm{E}-07$ & $2 \mathrm{E}-06$ & $2 \mathrm{E}-06$ & 63743 \\
\hline
\end{tabular}

Table 2: The results for time, $t=0.08$

\begin{tabular}{|c|c|c|c|c|c|c|c|}
\hline \multicolumn{2}{|c|}{$u(x, t) \backslash x$} & 0 & 0.2 & 0.4 & 0.6 & 0.8 & 1 \\
\hline \multirow{2}{*}{\multicolumn{2}{|c|}{ FDM }} & & 0.1851 & 0.3703 & 0.5555 & 0.7408 & \\
\hline & & 0 & 9 & 79 & 69 & 42 & 1 \\
\hline \multirow{2}{*}{\multicolumn{2}{|c|}{ DQM }} & & 0.1851 & 0.3703 & 0.5555 & 0.7407 & \\
\hline & & 0 & 85 & 7 & 56 & 42 & 1 \\
\hline \multirow{2}{*}{\multicolumn{2}{|c|}{ EXACT }} & & 0.1851 & 0.3703 & 0.5555 & 0.7406 & 0.9258 \\
\hline & & 0 & 69517 & 39035 & 08552 & 78069 & 47587 \\
\hline \multirow{4}{*}{$\begin{array}{l}\text { ER } \\
\text { RO } \\
\text { RS }\end{array}$} & \multirow{2}{*}{ FDM } & & 2.0482 & 3.9965 & 6.0448 & 0.0001 & 0.0741 \\
\hline & & 0 & $7 \mathrm{E}-05$ & $3 \mathrm{E}-05$ & E-05 & 63931 & 52413 \\
\hline & \multirow{2}{*}{ DQM } & & 1.5482 & 3.0965 & 4.7448 & 6.3930 & 0.0741 \\
\hline & & 0 & 7E-05 & $3 \mathrm{E}-05$ & E-05 & $6 \mathrm{E}-05$ & 52413 \\
\hline
\end{tabular}

From the Table 1 and 2, the errors between the result from FDM and DQM with the exact solution have been plotted in the graph that is depicted in Figure 2 and 3.

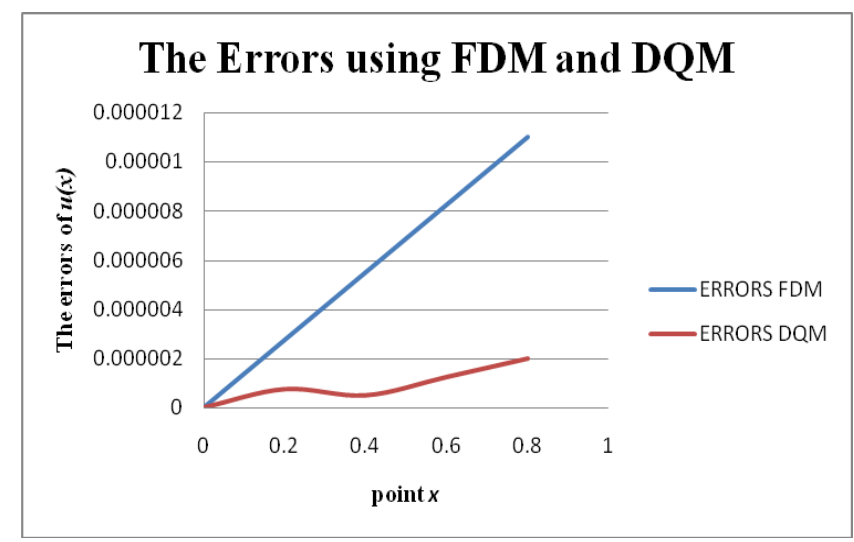

Figure 2: $t=0.04$

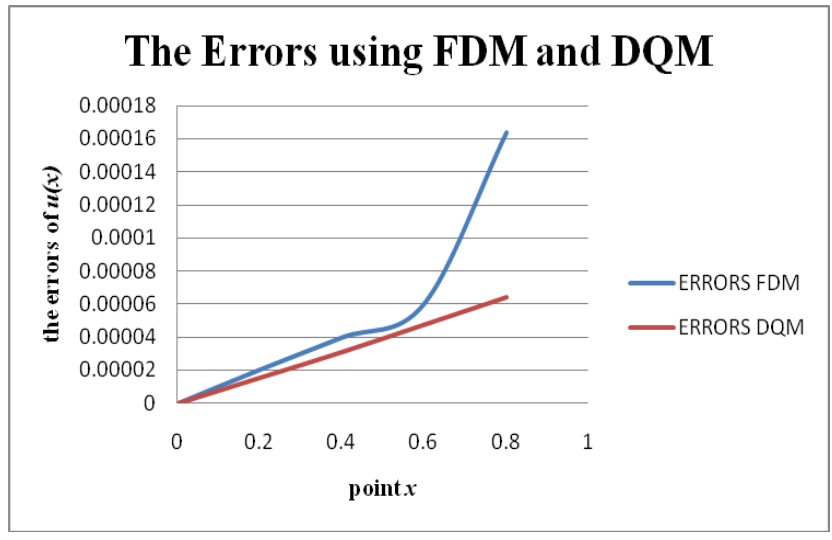

Figure 3: $t=0.08$

\subsection{CONCLUSION}

As a conclusion, from the tables and figures above, the results from the DQM showed better than FDM at each of time tested. In term of errors, the errors from the DQM results are smaller than the results from the FDM. Therefore, the DQM is better than FDM in term of the accurate. In term of convergent study, the results from DQM show faster convergent to the actual result comparing to FDM.

\section{REFERENCE}

1. Amiruddin Ab. Aziz, Hoe, Y.S., Iskandar, H. and Bahari, A.R. (2016). Application of Finite Difference Method and Differential Quadrature Method in Burgers Equation. Journal of Applied Environmental and Biological Sciences. 6(11), 111-114.

2. Çiçek, Y. and Tanoğlu, G. (2016). Strang Splitting Method for Burgers-Huxley Equation. Applied Mathematics and Computation. 276, 454-467.

3. Sari, M. and Gurarslan, G. (2009). Numerical Solutions of the Generalized Burgers-Huxley Equation by A Differential Quadrature Method. Mathematical Problems in Engineering. 2009, 370765.

4. Wazwaz, A.M. (2009). Burgers, Fisher and Related Equations. In Partial Differential Equations and Solitary Waves Theory. Nonlinear Physical Science. Springer, Berlin, Heidelberg

5. Yefimova, O.Y. and Kudryashov, N.A. (2004). Exact Solutions of the Burgers-Huxley Equation. Journal of Applied Mathematics and Mechanics 68(3), 413-420.

6. Gao, H. and Zhao, R.X. (2009). New Exact Solutions to the Generalized Burgers-Huxley Equation. Applied Mathematics and Computer.

7. Shu, C. (2000). Differential Quadrature and Its Application in Engineering. Great Britain: Springer-Verlag London.

8. Mittal, R.C. and Jiwari, R. (2009). Numerical Study of Burgers-Huxley Equation by Differential Quadrature 
Method. International Journal of Applied Math and Mechanic. 5(8), 1- 9.

9. Ngarisan, N.S. (2016). The Application of Finite Element Method to Solve Heat Transfer Problem Involving 2D Irregular Geometry. Journal of Applied Environmental and Biological Sciences. 6(11S), 23-30.

10. Chen, W. (1996). Differential Quadrature Method and its Applications in Engineering-Applying Special Matrix Product to Nonlinear Computations and Analysis. Ph.D. Thesis. Shanghai Jiao Tong University.

11. Palmer, A.C. and King,R.A. (2008). Subsea Pipeline Engineering: Pennwell Corporation.

12. Bai, Y. and Bai, Q. (2010). Subsea Engineering Handbook: Elsevier.

13. Shao, B., Yan, X. (2011). Reliability Analysis of Locally Thinned Submarine Pipelines in Cheng Dao Oil Field. Applied Mechanics and Materials. 94-96, 1527-1530.

14. Li, Z.G (2010). Configuration of Submarine Pipeline for Deep water S-Lay Technique. The International Society of Offshore and Polar Engineers. 